Tohoku J. Exp. Med., 2006, 208, 41-48

\title{
Atopy in Patients with Bronchiectasis: More than Coincidence
}

\author{
Sami Ozturk, Ergun Tozkoparan, ${ }^{1}$ Mehmet Karaayvaz, Zafer Caliskaner, \\ Mustafa Gulec, Omer Deniz, ${ }^{1}$ Ergun Ucar, ${ }^{1}$ Fatih $\mathrm{Ors}^{2}$ and Ugur Bozlar ${ }^{2}$ \\ Department of Allergic Diseases, ${ }^{\prime}$ Department of Pulmonary Medicine, \\ ${ }^{2}$ Department of Radiology, Gulhane Military Medical Academy, Ankara, Turkey
}

\begin{abstract}
Ozturk, S., Tozkoparan, E., Karaayvaz, M., Caliskaner, Z., Gulec, M., Deniz, O., Ucar, E., Ors, F. and Bozlar, U. Atopy in Patients with Bronchiectasis: More than Coincidence. Tohoku J. Exp. Med., 2006, 208 (1), 41-48 — Bronchiectasis is common in developing countries, but its precise underlying mechanism can be detected in only about $40 \%$ of the cases. The studies reporting the frequency of atopy and its relation to radiological findings and lung function in bronchiectasis are limited in number, and the results are controversial. The present study was designed to investigate the relationship between atopy and bronchiectasis by means of high resolution computed tomography (HRCT) and pulmonary function tests. Skin prick test, HRCT and pulmonary function tests, including spirometric values of forced expiratory volume in one second (FEV1), FEV1/FVC (forced vital capacity) ratio were performed in 121 bronchiectatic patients of unknown etiology and in 68 healthy controls. Atopy and HRCT scores for the severity of atopy and extent of bronchiectasis respectively were determined for each patient. The rate of atopy (48.8\% vs $11.8 \%)$ and mean atopy score $(14.3 \pm 10.1 \mathrm{~mm}$ vs $5.5 \pm 2.1 \mathrm{~mm})$ were significantly higher in patients with bronchiectasis than those in controls. Atopic patients had significantly worse spirometric values and more extended bronchiectasis than non-atopics. There is a significant correlation between atopy and HRCT scores $(r=0.54, p<0.001)$, indicating that the more severe atopy is the more extended bronchiectasis. In conclusion, we suggest that the rate of atopy is higher in bronchiectatic patients than that in healthy controls. Bronchiectatic patients with atopy have lower spirometric values and higher HRCT scores. Atopy might be considered as a deteriorating and/or a causative or contributing factor for development of bronchiectasis. —— bronchiectasis; atopy; high-resolution computed tomography; pulmonary function

(C) 2006 Tohoku University Medical Press
\end{abstract}

Bronchiectasis is defined pathologically as abnormal and permanent dilatation of the bronchial tree. Although marked an "orphan" disease in western countries (Barker and Bardana 1988), it is not uncommon in developing countries and remains a common cause of significant morbidity and mortality. In fact bronchiectasis is not a dis- ease per se, but it is an end stage manifestation of a variety of pathologic processes seen in different diseases (Cohen and Sahn 1999). The precise underlying mechanism can be detected in only about $40 \%$ of the patients and a minority of patients $(15 \%)$ benefit from specific treatment (Tsang and Tipoe 2004). High-resolution com-

Received July 19, 2005; revision accepted for publication October 19, 2005.

Correspondence: Dr. Sami Ozturk, GATA Allerjik Hastaliklar BD, Etlik TR-06018, Ankara, Turkey.

e-mail: samiozturkiz@yahoo.com, samiozturkiz@hotmail.com 
puted tomography (HRCT) is the diagnostic tool of choice and may also contribute to clinical management of the disease. Although less severe forms of bronchiectasis can be documented with the increased use of HRCT, it usually remains to be insufficient to demonstrate underlying cause.

Although an increased prevalence of reversible airway obstruction, bronchial hyperreactivity, atopic diseases such as asthma has been reported in patients with bronchiectasis (Varpela et al. 1978; Murphy et al. 1984; Pang et al. 1989; Ip et al. 1991, 1992; Hassan et al. 1999), the results of the studies in the literature investigating the frequency of atopy in bronchiectasis are a few in number and controversial (Varpela et al. 1978; Murphy et al. 1984; Pang et al. 1989). Furthermore, to our knowledge, there are no data in the literature about the relation of atopy with lung function and radiologic extent on HRCT in patients with bronchiectasis.

In view of these conflicting data, we aimed to investigate prospectively the frequency of atopy to inhaled allergens and its relationship to the extent and severity of the disease on HRCT and pulmonary function in patients with bronchiectasis.

\section{Materials and Methods}

\section{Subjects}

This study was conducted at Department of Pulmonary Medicine and Department of Allergic Diseases of Gulhane Military Medical Academy (GMMA) in Ankara. GMMA is a teaching hospital that serves as referral center for Turkish Armed Forces and primary care facility in Ankara metropolitan area. From September 1, 2002 to December 31, 2004, all consecutive patients with bronchiectasis admitted to our departments were enrolled to the study. Patients with any known cause of bronchiectasis including cystic fibrosis, established diagnosis of asthma and allergic bronchopulmonary aspergillosis, previous tuberculosis, humoral immunodeficiency, autoimmune and collagen-vascular diseases and $\alpha_{1}$-antitrypsin deficiency were excluded. Finally 121 patients with bronchiectasis without any known etiology were included. Patients' characteristics including age, sex, smoking history, disease duration, history of familial atopy, childhood and current allergic disease were recorded. Since patients with bronchial asthma, a potential cause of bronchiectasis, were excluded, current allergic disease included atopic dermatitis and allergic rhinitis. Sixty-eight healthy subjects who are free of any allergic disease such as asthma and allergic rhinitis, served as controls. An informed consent was obtained from all study subjects. The study was approved by the local ethics committee of GMMA.

\section{Diagnostic criteria and HRCT scoring}

In all patients, HRCT scans with $1 \mathrm{~mm}$ collimation at $10 \mathrm{~mm}$ intervals were obtained through the thorax at end inspiration and they were interpreted by two radiologists who were blinded to clinical history and chest $\mathrm{x}$-ray findings. Criteria for the diagnosis of bronchiectasis utilizing HRCT included the following: 1) dilatation of bronchi as determined by an increased bronchial/adjacent pulmonary artery ratio (ratio $>1$ ) when imaged in cross section (signet-ring sign), 2) parallel bronchial walls when seen in longitudinal section (tram sign), and 3) demonstration of bronchi in the peripheral third of the lung (Pifferi et al. 2004). Bronchiectasis was scored as previously described (Twiss et al. 2005) using the modified Bhalla scoring system (Bhalla et al. 1991; Roberts et al. 2000). This score has been validated in adult and pediatric patients with bronchiectasis and assigns a value to each lobe and the lingula as follows: bronchiectasis extent (0-3), bronchial wall dilatation (0-3) and thickness $(0-3)$, presence of mucus in large (0-1) and small airways (0-1), air trapping (0-4), atelectasis (0-1) and consolidation (0-1) resulting in a worst possible score of 102 (Roberts et al. 2000). The higher HRCT score represented the more extended and severe bronchiectasis.

\section{Spirometry}

Lung function measurements were performed using a spirometer (Sensormedics 2400, Sensormedics Company, Bilthoven, Netherlands) according to the standardized procedures of the European Respiratory Society. Volume calibration of the spirometer was done before each measurement. The forced vital capacity (FVC) and forced expiratory volume in one second (FEV1) were taken as the highest readings obtained from at least three satisfactory forced expiratory maneuvers. The participants' baseline lung function was compared with the reference values of Knudson et al. (1976) and expressed as percent of the predicted values.

\section{Skin prick test}

Skin prick tests (SPT) were performed by allergists at the department of Allergic Disease of GMMA accord- 
ing to a routine procedure and using a standard panel of 56 common aeroallergens including mites (D. farinea and D. pteronyssinus), molds, grass mix, tree mix pollen, weed mix, cockroach and animal dander (Greer Lab, NC, USA). Drugs that depress the immediate type skin tests such as antihistamines or tricyclic antidepressants were discontinued 7 days prior to the test. SPT were applied on the volar surface of the forearm by the same investigator using disposable lancets and reactions were recorded 15 min after the test. Resulting wheal diameter larger than $3 \mathrm{~mm}$ with surrounding erythema was considered as positive for atopy if the positive control histamine was positive and negative control was negative. In order to differentiate dermographic and irritant reactions, test with negative control solution (diluent with $0.9 \%$ saline and $0.4 \%$ phenol) was particularly performed to all subjects. The wheal without erythema was accepted as irritant reaction and was excluded. An atopy score for the degree of atopy was calculated as sum of mean wheal diameter of 56 allergens tested, as previously defined
(Koh et al. 2002). The higher atopy score was considered as having the more severe atopy.

\section{Statistical analysis}

SPSS (SPSS for Windows, SPSS Inc., Chicago, IL, USA) statistical package were used for statistical analyses. Descriptive statistics were shown as mean \pm S.D. Univariate analysis was performed using chi-square test for proportion of atopy, sex differences in the study and control groups. To compare parametric values of the groups including age, disease duration, mean HRCT and atopy scores and lung function parameters, Student's $t$-test was used. Correlation of the atopy score with lung function parameters and HRCT score was investigated by Pearson correlation test. A $p$ value less than 0.05 was considered statistically significant.

\section{Results}

Characteristics of the study population were shown in Table 1. Since the study was conducted

TABLE 1. Characteristics of the study population

\begin{tabular}{lcc}
\hline & Bronchiectasis $(n=121)$ & Control $(n=68)$ \\
\hline Sex $(\mathrm{M} / \mathrm{F})$ & $113 / 8^{\#}$ & $60 / 8$ \\
Mean age, years (range) & $24.6 \pm 6.7^{\#}(18-43)$ & $24.7 \pm 4.7(18-41)$ \\
Smoking history & $54^{\#}$ & 29 \\
Non-smoker & $48^{\#}$ & 22 \\
Ex-smoker & $19^{\#}$ & 17 \\
Current-smoker & $59(48.8 \%)^{*}$ & $8(11.8 \%)$ \\
Atopic subjects $(\%)$ & $47(39 \%)^{*}$ & $4(6 \%)$ \\
House dust mite & $16(13 \%)^{\#}$ & $3(4 \%)$ \\
Grass mix & $10(8 \%)^{\#}$ & $2(3 \%)$ \\
Tree mix & $10(8 \%)^{* *}$ & 0 \\
Weed mix & $7(6 \%)^{\#}$ & 0 \\
Mold mix & $10(8 \%)^{\#}$ & $1(1 \%)$ \\
Cockroach & 0 & 0 \\
Animal dander & $25.9 \pm 12.8$ & - \\
HRCT score & $14.3 \pm 10.1^{*}$ & $5.5 \pm 2.1$ \\
Atopy score & $67.7 \pm 11.9^{*}$ & $105.3 \pm 8.9$ \\
FEV1 (\% predicted) & $70.1 \pm 8.6^{*}$ & $105.6 \pm 7.0$ \\
FEV1/FVC $(\%$ predicted) & &
\end{tabular}

${ }^{\#}$ Not differed significantly.

${ }^{*}$ Significantly differed from controls $(p<0.001)$.

${ }^{* *}$ Significantly differed from controls $(p=0.036)$.

${ }^{\S}$ Per atopic subject. 
TABLE 2. Data of the atopic and non-atopic patients with bronchiectasis

\begin{tabular}{lccc}
\hline & $\begin{array}{c}\text { Atopics } \\
(n=59)\end{array}$ & $\begin{array}{c}\text { Non-atopics } \\
(n=62)\end{array}$ & Significance \\
\hline Sex (M/F) & $54 / 5$ & $59 / 3$ & Not significant \\
Mean age, years & $24.3 \pm 4.4$ & $24.8 \pm 5.0$ & Not significant \\
Smoking history & & & \\
$\quad$ Non-smoker & 25 & 29 & Not significant \\
$\quad$ Ex-smoker & 27 & 21 & Not significant \\
$\quad$ Current-smoker & 7 & 12 & Not significant \\
Duration of bronchiectasis, years & $6.2 \pm 4.3$ & $4.6 \pm 3.5$ & $p=0.023$ \\
Familial Atopy, $\mathrm{n}$ & $15(\% 25)$ & $5(\% 8)$ & $p=0.020$ \\
Childhood allergic disease, $\mathrm{n}$ & $16(\% 27)$ & $6(\% 10)$ & $p=0.024$ \\
Current allergic disease, $\mathrm{n}$ & $18^{*}(\% 31)$ & $8^{\#}(\% 13)$ & $p=0.033$ \\
HRCT score & $33.3 \pm 12.1^{*}$ & $18.8 \pm 8.8$ & $p<0.001$ \\
FEV1 (\% predicted) & $62.7 \pm 10.7^{*}$ & $72.6 \pm 10.8$ & $p<0.001$ \\
FEV1/FVC $(\%$ predicted $)$ & $66.2 \pm 7.8^{*}$ & $73.9 \pm 7.6$ & $p<0.001$ \\
\hline
\end{tabular}

* two atopic dermatites and 16 allergic rhinitis.

\# one atopic dermatitis and 7 allergic rhinitis.

in a military hospital, most of the patients were male but demographic characteristics and smoking habits of the groups did not show a significant difference. In 121 patients with bronchiectasis, whose mean age was $24.6 \pm 6.7$ and of whom 113 were male, $59(48.8 \%)$ patients were assessed as atopic while only $8(11.8 \%)$ of the 68 controls were atopic $(p<0.001)$. The most common allergen was house dust mites (39\%). The frequencies of positive results with other allergens including grass pollen, tree pollen, weed pollen, cockroach and molds among patients with bronchiectasis were $13 \%, 8 \%, 8 \%, 8 \%$ and $6 \%$, respectively. When patients with bronchiectasis and the controls were compared according to specific allergen sensitivity, patients with bronchiectasis had significantly more positive reactions to house dust mites and weed mix than controls $(p<0.001$ and $p=0.036$, respectively) while sensitivity to other allergens did not differ significantly between the groups. Mean atopy score (per atopic subject) was significantly higher in patients with bronchiectasis $(14.3 \mathrm{~mm} \pm 10.1 ; p<0.001)$ than in controls $(5.5 \pm 2.1 \mathrm{~mm})$. Study group had also significantly diminished pulmonary function with respect to controls.
Mean HRCT score was significantly higher in atopic patients $(33.3 \pm 12.1)$ with bronchiectasis than in those nonatopic $(18.8 \pm 8.8)$ (Table 2). Atopic patients had also significantly worse FEV1 $(62.7 \pm 10.7 \% ; p<0.001)$ and FEV 1/FVC $(66.2 \pm$ $7.8 \%$ ) values, longer disease duration, frequent history of familial atopy, childhood and current allergic disease than non-atopics. Smoking habit did not differ significantly between atopics and non-atopics.

Atopy score was correlated positively with HRCT score $(r=0.54$ and $p<0.001)$ and negatively with FEV1 $(r=-0.49$ and $p<0.001)$ and FEV1/FVC $(r=-0.49$ and $p<0.001)$ values (Fig. 1-3).

\section{DiscuSSION}

This study has demonstrated that atopy is more frequent in patients with bronchiectasis than in healthy individuals. Atopic patients with bronchiectasis had significantly worse pulmonary function and higher HRCT score, which was indicated more extended and severe bronchiectasis than their non-atopic counterparts. Furthermore atopy score was correlated with positively with HRCT score and negatively with pulmonary func- 


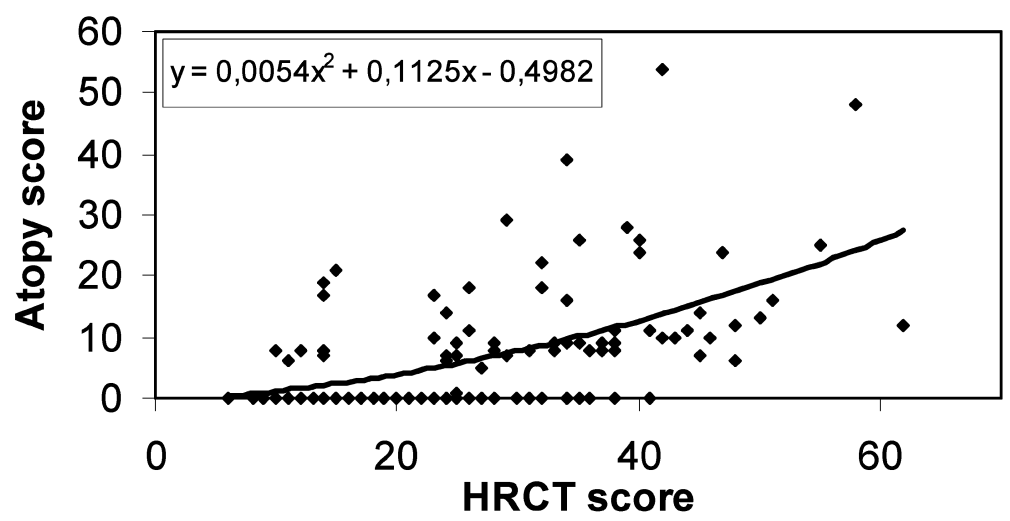

Fig. 1. Correlation of atopy and HRCT scores.

The atopy scores of the nonatopic subjects were accepted as zero. $r=0.54$ and $p<0.001$.

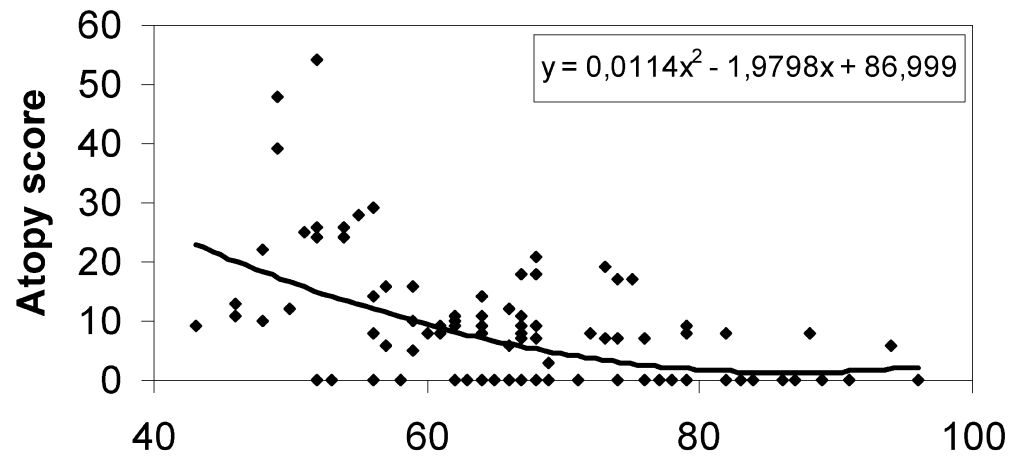

FEV1 \%

Fig. 2. Correlation of atopy score and FEV1.

The atopy scores of the nonatopic subjects were accepted as zero. FEV1 \%: Percent of the predicted value. $r=-0.49$ and $p<0.001$.

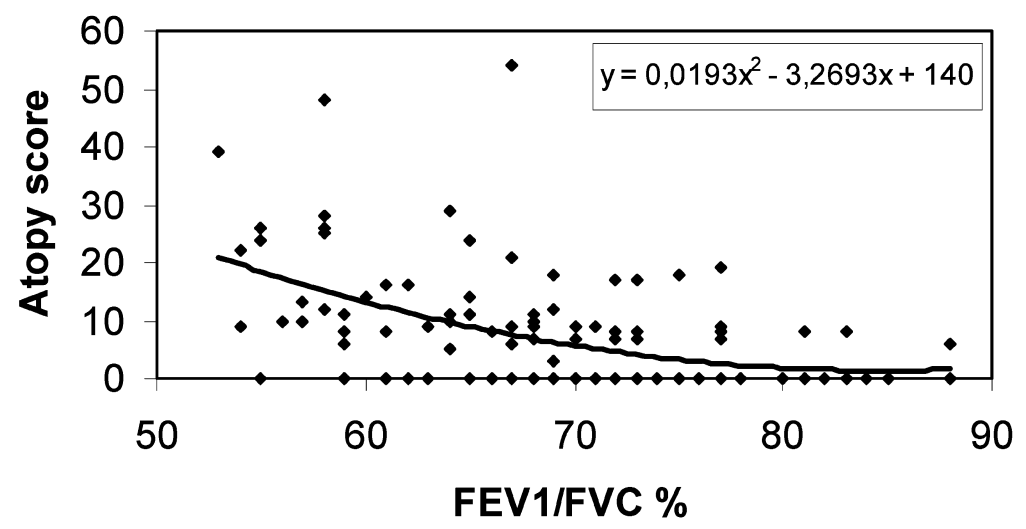

Fig. 3. Correlation of atopy score and FEV1/FVC.

The atopy scores of the nonatopic subjects were accepted as zero. $r=-0.49$ and $p<0.001$. 
tion parameters of FEV1 and FEV1/FVC. These findings indicate that the more severe atopy results in the worse pulmonary function and the more extended and severe bronchiectasis. House dust mites was the major allergen among bronchiectatic patients and atopic patients had longer disease duration than those non-atopics.

Bronchiectasis is a disease that no cause can be defined in about $40 \%$ of the cases (Tsang and Tipoe 2004). It is characterized generally by bronchial obstruction and hyperreactivity of which the mechanisms, however, are not well understood (Varpela et al. 1978; Pang et al. 1989; Ip et al. 1991; Hassan et al. 1999). It has been suggested that mucosal edema, glandular hyperplasia, excessive airway collapse in expiration might result in bronchial obstruction (Hassan et al. 1999). Association of atopy and allergic diseases such as asthma and alteration of bronchial smooth muscle and autonomic neural regulation due to increased access to toxins through infected or inflamed bronchial mucosa could cause bronchial hyperreactivity (Pang et al. 1989). It has been suggested that coexistence of asthma with bronchiectasis alone cannot explain bronchial hyperreactivity and obstruction (Ip et al. 1991). According to our results, lower pulmonary function in atopic bronchiectatic patients than nonatopics ones suggests that atopy may have a role in bronchial obstruction in bronchiectasis.

The results of the studies in the literature investigating the prevalence of atopy in bronchiectasis are a few in number and conflicting (Varpela et al. 1978; Murphy et al. 1984; Pang et al. 1989; Hassan et al. 1999). Varpela et al. (1978) found prevalence of atopy in their series of 48 patients with bronchiectasis as $10 \%$. In the study of Hassan et al. (1999), SPT yielded a positive result in 13 of 24 (54\%) patients with bronchiectasis while Pang et al. (1989) and Murphy et al. (1978) reported atopy prevalence in bronchiectasis as 25 and $30 \%$, respectively.

The studies yielding results different from ours have methodological differences. The study of Varpela et al. (1978) was uncontrolled and diagnosis of bronchiectasis was based on bronchography, which is less sensitive than HRCT.
SPT was carried out with only 11 allergens in the study of Pang et al. (1989). Although Murphy et al. (1978) reported that no increased prevalence of atopy in patients with bronchiectasis compared to healthy controls, patients with positive SPT results showed a larger mean wheal diameter (mean atopy score) than controls with positive responses and they used bronchography to diagnose bronchiectasis instead of HRCT. Although findings of Hassan et al. (1999) support our results, their study was uncontrolled. Our study population was larger than those mentioned above and significant correlations of atopy score with HRCT score and lung function have been reported for the first time in the literature. The method for atopy scoring needs to be validated and standardized in further studies. The male predominance of our study may constitute another limitation of the study but we suppose that these limitations do not invalidate our findings. Furthermore we must admit that the atopy prevalence of our control group is lower than that of Turkish Population reported in previous studies (Celik et al. 1999). In their study which is one the most comprehensive studies investigating prevalence of atopy and allergic disease in Turkish Population. Celik et al. (1999) reported atopy prevalence of Turkish population as $25 \%$ and $80 \%$ of the atopic Turkish subjects had an allergic disease such as asthma and/or allergic rhinitis. Since the subjects of our control group are healthy and have no associated allergic disease, the group does not represent the Turkish Population and the atopy prevalence of the group would be expected to be lower than that of the whole population.

One may have thought that our results are in conflict with hygiene hypothesis with regard to development of atopy and allergic diseases since recurrent respiratory infections in bronchiectasis would give rise to expect a decreased prevalence of atopy. Though, we suppose that our study do not contradict the hygiene hypothesis and association of bronchiectasis and atopy can be explained from several points of view. Firstly, some infections that may be associated with both atopy and bronchiectasis such as measles, pertussis and staphylococcal infections might have taken part in 
development of atopy and bronchiectasis at the same time, as does allergic bronchopulmonary aspergillosis. It has been reported that pertussis, a causative factor for bronchiectasis (Pasteur et al. 2000; Tsang and Tipoe 2004), is associated with subsequent atopy and atopic disorders such as asthma and eczema (Bodner et al. 1998; Farooqi and Hopkin 1998; Al-Mousawi et al. 2004). Another childhood infection, which is a significant causative factor of bronchiectasis (Pasteur et al. 2000; Tsang and Tipoe 2004) and may be associated with atopy, is measles although controversies exist with regard to its relationship with atopy and atopic diseases. In a very large Finnish study, which included half a million subjects aged 14 months to 19 years, Paunio et al. (2000) reported that measles and atopy had occurred more frequently together than expected. Furthermore Bager et al. (2002) reported that Danish women with measles during the first year of life was associated with a 3.4 fold increase in the odds ratio of atopy compared to women without measles before the age of 7 years, while other investigations revealed that either measles had a protective effect against atopic disorders or there was no relationship between them (Farooqi and Hopkin 1998; von Mutius 2001). The controversy about this issue may be explained by biphasic response of natural measles infection; in the early phase T-helper (Th)-1 type immune response with increased levels of interleukin (IL)-12 is a characteristic feature, while in the later phase a prolonged Th-2 type response with increased levels of IL-4 and Ig-E predominates which subsequently favors the development of atopic conditions (von Hertzen and Haahtela 2004). Staphylococcus infection, which is another narcotizing lung disease and a significant cause of bronchiectasis (Tsang and Tipoe 2004) can induce IL-5 stimulation and play a role as a cofactor in the pathogenesis of atopic disease (Heaton et al. 2003). A role of staphylococcal superantigens in atopic dermatitis has been defined and similar mechanisms might be relevant in airway diseases including asthma (Bachert et al. 2002; Suh et al. 2004).

Secondly, coincidence of bronchiectasis and some other infections such as hepatitis B virus
(HBV) infection that can potentially induce atopy may be another explanation of association of bronchiectasis and atopy. The prevalence of HBV infection is relatively higher in countries where bronchiectasis is common. Th-1 response in chronic HBV carriers is insufficient; as a result, the Th-2 response may develop, and an increased tendency to develop atopy and allergic diseases (Kocabas 2001). Deficiency of Th1 response to HBV with decreased levels of IL-12 and interferon- $\gamma$ is demonstrated in chronic HBV infection (Naumov and Rossol 1997; Ji et al. 2001).

Thirdly, different inflammatory response to infections and high incidence of comorbidity with bacterial infections including sinusitis (Mucha and Baroody 2003) in atopic subjects may be a contributing factor in pathogenesis of bronchiectasis. It has been reported that virus-induced inflammatory changes in upper respiratory tract infections are more prolonged in atopic than in nonatopic subjects (Corne et al. 2001), suggesting that it might have resulted in higher probability of bronchiectasis. Another interesting study revealed that elevated concentrations of serum amyloid-A in patients with allergic airway disease might reflect presence of a systemic inflammation (Buyukozturk et al. 2004).

Since these are hypothetical explanations with regard to association of atopy and bronchiectasis, further studies based on epidemiological and immunological evidences are needed to unravel the exact mechanism of the issue. However we suppose that atopy may be a causative or contributing effect on development of bronchiectasis, as well as a coincidence, since the incidence of bronchiectasis can be higher than expected in countries where atopy prevalence is also high (Twiss et al. 2005). Furthermore atopy might be deteriorating factor for bronchiectasis since atopy score has been positively correlated with HRCT score, in other words, the more atopy is the more extended bronchiectasis.

In conclusion, we suggest that atopy is significantly frequent in bronchiectasis and it may lead to worse pulmonary function and more radiologic extent in patients with bronchiectasis. Clinicians should be aware of this issue, however 
exact relationship with these entities, remains to be investigated by means of epidemiological and immunological studies.

\section{References}

Al-Mousawi, M.S.H., Lovel, H., Behbehani, N., Arifhodzic, N., Woodcock, A. \& Custovic, A. (2004) Asthma and sensitization in a community with low indoor allergen levels and low pet-keeping frequency. J. Allergy Clin. Immunol., 114, 1389-1394.

Bachert, C., Gevaert, P. \& van Cauwenberge, P. (2002) Staphylococcus aureus superantigens and airway disease. Curr. Allergy Asthma Rep., 2, 252-258.

Bager, P., Westergaard, T., Rostgaard, K., Hjalgrim, H. \& Melbye, M. (2002) Age at childhood infections and risk of atopy. Thorax, 57, 379-382.

Barker, A.F. \& Bardana, E.J., Jr. (1988) Bronchiectasis: update of an orphan disease. Am. Rev. Respir. Dis., 137, 969-978.

Bhalla, M., Turcois, N., Aponte, V., Jenkins, M., Leitman, B.S., McCauley, D.I. \& Naidich, D.P. (1991) Cystic fibrosis: scoring system with thin section CT. Radiology, 179, 783-788.

Bodner, C., Godden, G. \& Seaton, A. (1998) Family size, childhood infections and atopic diseases. Thorax, 53, 28-32.

Büyüköztürk, S., Gelincik, A.A., Genç, S., Kocak, H., Öneriyidogan, Y., Erden, S., Dal, M. \& Çolakoglu, B. (2004) Acute phase reactants in allergic airway disease. Tohoku J. Exp. Med., 204, 209-213.

Celik, G., Mungan, D., Bavbek, S., Sin, B., Ediger, D., Demirel, Y. \& Misirligil, Z. (1999) The prevalence of allergic diseases and atopy in Ankara, Turkey: A two-step population based epidemiological study. J. Asthma, 36, 281-290.

Cohen, M. \& Sahn, S.A. (1999) Bronchiectasis in systemic diseases. Chest, 116, 1063-1074.

Corne, J.M., Lau, L., Scott, S.J., Davies, R., Johnston, S.L. \& Howart, P.H. (2001) The relationship between atopic status and IL-10 nasal lavage levels in the acute and persistent inflammatory response to upper respiratory tract infection. Am. J. Respir. Crit. Care Med., 163, 1101-1107.

Farooqi, I.S. \& Hopkin, J.M. (1998) Early childhood infection and atopic disorder. Thorax, 53, 927-932.

Hassan, J.A., Saadiah, S., Roslan, H. \& Zainudin, B.M.Z. (1999) Bronchodilator response to inhaled beta-2 agonist and anticholinergic drugs in patients with bronchiectasis. Respirology, 4, 423-426.

Heaton, T., Mallon, D., Venaille, T. \& Holt, P. (2003) Staphylococcal enterotoxin induced IL-5 stimulation as a cofactor in the pathogenesis of atopic disease: the hygiene hypothesis in reverse? Allergy, 58, 252-256.

Ip, M., Lam, M.W., So, S.Y., Liong, E., Chan, C.Y. \& Tse, K.M. (1991) Analysis of factors associated with bronchial hyperreactivity to methacholine in bronchiectasis. Lung, 169, 43-51.

Ip, M.S.M., So, S.Y., Lam, W.K. \& Liong, E. (1992) High prevalence of asthma in patients with bronchiectasis in Hong Kong. Eur. Respir. J., 5, 418-423.

Ji, W., Wang, H.F. \& Feng, C.Q. (2001) Activation-induced cell death in peripheral blood mononuclear cells (PBMCs) from patients with chronic hepatitis B may be related to abnormal production of interleukin 12 and 10. J. Viral Hepat., 8,
30-33.

Knudson, R.J., Slatin, R.C., Lebowitz, M.D. \& Burrows, B. (1976) The maximal expiratory flow volume curve: normal standards, variability, and effects of age. Am. Rev. Respir. Dis., 113, 587-600.

Kocabas, C.N. (2001) Do hepatitis B virus carriers develop atopic diseases? Allergy, 56, 1100-1101.

Koh, Y.I., Choi, I.S. \& Lim, H. (2002) Atopy may be related to exercise-induced bronchospasm in asthma. Clin. Exp. Allergy, 32, 532-536.

Mucha, S.M. \& Baroody, F.M. (2003) Relationships between atopy and bacterial infections. Curr. Allergy Asthma Rep., 3, 232-237.

Murphy, M.B., Reen, D.J. \& Fitzgerald, M.X. (1984) Atopy, immunological changes, and respiratory function in bronchiectasis. Thorax, 39, 179-184.

Naumov, N.V. \& Rossol, S. (1997) Studies of interleukin-12 in chronic hepatitis B virus infection. J. Viral Hepat., 4 Suppl, 87-91.

Pang, J., Chan, H.S. \& Sung, J.Y. (1989) Prevalence of asthma, atopy, and bronchial hyperreactivity in bronchiectasis: a controlled study. Thorax, 44, 948-951.

Pasteur, M.C., Helliwell, S.M., Houghton, S.J., Webb, S.C., Foweraker, J.E., Coulden, R.A., Flower, C.D., Bilton, D. \& Keogan, M.T. (2000) An investigation into causative factors in patients with bronchiectasis. Am. J. Respir. Crit. Care Med., 162, 1277-1284.

Paunio, M., Heinonen, O.P., Virtanen, M., Leinikki, P., Patja, A. \& Peltola, H. (2000) Measles history and atopic diseases: a population-based cross-sectional study. JAMA, 283, 343-346.

Pifferi, M., Caramella D., Bulleri, A., Baldi, S., Peroni, D., Pietrobelli A. \& Boner, A.L. (2004) Pediatric bronchiectasis: correlation of HRCT, ventilation and perfusion scintigraphy, and pulmonary function testing. Pediatr. Pulmonol., 38, 298-303.

Roberts, H.R., Wells, A.U., Milne, D.G., Rubens, M.B., Kolbe, J., Cole, P.J. \& Hansell, D.M. (2000) Airflow obstruction in bronchiectasis: correlation between computed tomography features and pulmonary function tests. Thorax, $\mathbf{5 5}$, 198-204.

Suh, Y.J., Yoon, S.H., Sampson, A.P., Kim, H.J., Kim, S.H., Nahm, D.H., Suh, C.H. \& Park, H.S. (2004) Specific immunoglobulin $\mathrm{E}$ for staphylococcal enterotoxins in nasal polyps from patients with aspirin-intolerant asthma. Clin. Exp. Allergy, 34, 1270-1275.

Tsang, K.W. \& Tipoe, G.L. (2004) Bronchiectasis: not an orphan disease in the East. Int. J. Tuberc. Lung Dis., 8, 691-702.

Twiss, J., Metcalfe, R., Edwards, E. \& Byrnes, C. (2005) New Zealand national incidence of bronchiectasis "too high" for a developed country. Arch. Dis. Child., 90, 737-740.

Varpela, E., Laitinen, L.A., Keskinen, H. \& Korhola, O. (1978) Asthma, allergy and bronchial hyper-reactivity to histamine in patients with bronchiectasis. Clin. Allergy, 8, 273-280.

von Hertzen, L.C. \& Haahtela, T. (2004) Immunization and atopy: possible implication of ethnicity. J. Allergy Clin. Immunol., 113, 401-406.

von Mutius, E. (2001) Infection: friend or foe in the development of atopy and asthma? The epidemiological evidence. Eur. Respir. J., 18, 872-881. 